\title{
Faut-il aider les syndicats français ?
}

Le Débat, 142, novembre-décembre 2006, p. 119-132.

\author{
Dominique Andolfatto \\ Université de Bourgogne \\ (Dominique.Andolfatto@u-bourgogne.fr)
}

Dominique Labbé

PACTE (IEP de Grenoble)

(dominique.labbe@iep-grenoble.fr)

\section{Résumé}

Discussion de quatre idées reçues : la faiblesse historique de la syndicalisation française, l'infidélité des adhérents, la pauvreté des syndicats français et l'insuffisance du dialogue social. Ces prémisses fausses servent à justifier l'organisation d'élections de représentativité et de nouvelles aides publiques qui ne mettront pas fin à la désyndicalisation et risquent d'aggraver la crise syndicale.

\begin{abstract}
Discussion of four widespread beliefs: the historic weakness of the French trade unions, the infidelity of their members, the poverty of the French unions and the lack of social dialogue. These false premises lead to the organization of elections in order to measure the union representativeness and to the allocation of new public subsidies to these organizations. That will not end the de-unionization and may worsen the crisis of the French trade unionism.
\end{abstract}

Manuscrit de l'article paru dans : Le Débat, 142, novembre-décembre 2006, p. 119-132.

Toute citation doit se faire à partir de la revue. 
A propos du syndicalisme français, quatre idées reçues ont cours dans l'opinion, comme dans le monde syndical et même à l'université :

- les syndicats français n'ont jamais eu beaucoup d'adhérents, contrairement aux autres pays européens ;

- non seulement les adhérents sont peu nombreux, mais ils se montrent généralement volages et passifs. Ces individualistes ne font que passer quelques mois et se désintéressent du travail syndical qui repose sur quelques militants ;

- les syndicats français sont pauvres et dépourvus des moyens qui leur seraient nécessaires pour établir un dialogue équilibré et responsable avec le patronat et l'Etat.

- du fait de cette faiblesse structurelle, la négociation collective est peu développée en France. Cela freine la modernisation des administrations et des entreprises et cela rend difficile la réforme de l'Etat providence.

On tire de ces prémices une conclusion qui semble en passe de recueillir l'unanimité : puisque les syndicats sont indispensables à une économie moderne et que les Français ne leur apportent pas spontanément leur contribution, il faut que l'Etat finance ces organisations, comme il le fait pour les partis.

Les trois premières idées reçues ne résistent pas à un examen sérieux. La syndicalisation en France a été importante pendant plus d'une trentaine d'années (1945-1978). Deuxièmement, les adhérents ont été fidèles et une minorité significative a même apporté une contribution qui dépassait largement le simple paiement régulier de la cotisation. Troisièmement, depuis près de 40 ans, les syndicats français disposent de moyens considérables et en augmentation constante. Enfin, on peut s'interroger sur le prétendu blocage de la négociation collective. Dès lors, la conclusion - augmentation du financement public - ne semble guère fondée. Il y a même de sérieuses raisons de penser qu'elle serait contre-productive. 


\section{Faiblesse historique des syndicats français ?}

Il est exact qu'il y avait peu de syndiqués en France avant 1914 : moins de 800000 syndiqués dont 400000 à la CGT $^{1}$. Dès 1917 , une première vague d'adhésions porte la CGT à plus de 2 millions d'adhérents à la fin de 1919. Une autre vague plus considérable encore se produit en 1936, de telle sorte qu'en 1937-38, la moitié des salariés français sont syndiqués et que la CGT comporte à elle seule plus de 4 millions d'adhérents ${ }^{2}$.

Contrairement aux deux vagues précédentes, celle qui suit la Libération résiste mieux et, entre 1958 et 1978, plus du quart des salariés ont été syndiqués (graphique ci-dessous) avec, même, une nette poussée entre 1967 et 1977 : pendant ces dix années, pratiquement 3 salariés sur 10 étaient syndiqués ${ }^{3}$.

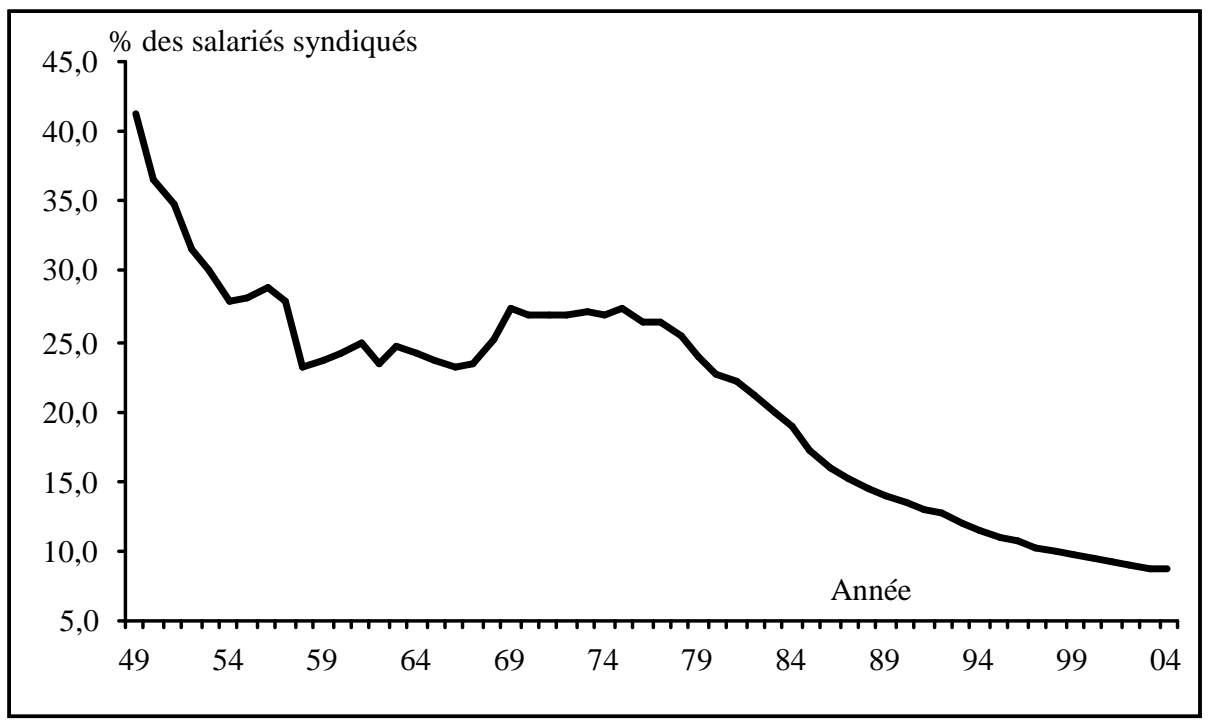

Durant ces années de croissance économique rapide, la population active salariée française est passée de 13 à 18 millions : le simple maintien du taux de syndicalisation implique donc un grand nombre d'adhésions supplémentaires. Autrement dit, durant

\footnotetext{
${ }^{1}$ Dominique Andolfatto et Dominique Labbé. Histoire des syndicats (1906-2006). Paris : Seuil, 2006.

${ }^{2}$ Antoine Prost. Autour du Front populaire : aspects du mouvement social au XXe siècle. Paris : Seuil, 2006.

${ }^{3}$ Ces données proviennent d'une enquête réalisée pour le ministère du travail en 1995 (synthèse dans : Dominique Labbé. Syndicats et syndiqués en France depuis 1945. Paris : l'Harmattan, 1996). Cette enquête est en cours d'actualisation sous la direction de Dominique Andolfatto (le rapport sera remis au Ministère du travail à l'automne 2006).
} 
les "trente glorieuses", le syndicalisme français a su s'adapter aux changements $\mathrm{du}$ salariat français : féminisation, tertiarisation, montée du travail qualifié ${ }^{4}$.

Certes le taux de syndicalisation était plus faible qu'en Allemagne et que dans les pays scandinaves ou en Belgique, mais il était comparable à celui d'Amérique du Nord et il était nettement supérieur à celui de l'Italie, du moins jusqu'au début des années 1970. La faible syndicalisation n'est donc pas une fatalité française comme on le dit habituellement ${ }^{5}$.

Dès lors, deux questions se posent : pourquoi y avait-il autant d'adhérents ? Pourquoi sont-ils partis?

Jusqu'à la fin des années 1970, la principale activité des militants syndicaux consistait à apporter aux adhérents une aide individuelle contre les licenciements, les sanctions, les mutations arbitraires, les brimades - celles des chefs mais aussi celles des collègues - et pour résoudre les multiples problèmes de la vie quotidienne au travail: mauvaises conditions de travail, machines dangereuses, horaires incommodes, congés refusés, primes non payées... Cette fonction de "défense et recours" n'était guère appréciée par les militants car elle dévorait leur temps, sans faire avancer les "luttes", mais elle était acceptée car la tournée du délégué du personnel (DP) était le moyen de récupérer les cotisations et parce que des interventions efficaces permettaient de gagner des adhérents et des électeurs. Cette activité se déroulait dans une coopération conflictuelle avec la maîtrise. Elle était source d'un droit coutumier régissant la vie quotidienne au travail d'une manière beaucoup plus souple et plus efficace que le code du travail ou que la convention collective. Une certaine culture ouvrière y était associée qui constituait un facteur décisif d'intégration des jeunes, des ruraux déracinés puis des immigrés.

Pendant plus de 30 ans, malgré tous leurs défauts, qu'il ne faut pas minimiser, les syndicats français ont semblé suffisamment intéressants aux yeux de millions de salariés pour qu'ils leur apportent au moins une cotisation régulière et renouvelée assez fidèlement. Parmi eux, une minorité significative a assuré des tâches bénévoles

\footnotetext{
${ }^{4}$ Pour une description synthétique de ce syndicalisme "à la française" : Dominique Labbé. Syndicats et syndiqués. Op. cit, p 58-73.

5 Par exemple, Thomas Amossé. «Mythes et réalités de la syndicalisation en France ». Premières synthèses. DARES, $\mathrm{n}^{\circ}$ 44-2, octobre 2004. L'auteur a réduit les effectifs syndiqués depuis 1945 de $20 \%$ en considérant que tel était le poids des retraités dans le mouvement syndical. En réalité, ce poids était négligeable, avant les années 1970 et ne représente encore aujourd'hui que 19\% des effectifs de la CGT et environ $13 \%$ du total des syndiqués.
} 
(confection et distribution de tracts, collectage des cotisations, distribution de la presse, tenue des permanences...).

A partir des années 1970, ce système disparaît progressivement et, depuis lors, la syndicalisation ne cesse de baisser. En moins de 30 ans, le taux de syndicalisation a été divisé par trois (de 27\% en 1976 à 9\% en 2004). En effet, il y a actuellement environ 2,2 millions de syndiqués en France (1,9 millions d'actifs) parmi les 22,5 millions de salariés français. A cela s'ajoute la division. En 1945, pour 4 fois plus de syndiqués, il y avait 2 confédérations (CGT et CFTC) ; actuellement, il y a 7 "centrales" - CGT, CFDT, FO, UNSA, CFTC, CGC-CFE, SUD - ou 8 si l'on y ajoute la $\mathrm{FSU}^{6}$.

Le noyau du syndicalisme français contemporain est fourni par les salariés à statut des grandes entreprises nationales (EDF, SNCF, RATP, Arsenaux) auxquels s'ajoutent les postiers et les employés de France-Télécom, les fonctionnaires de l'Education nationale, des Finances et de l'Equipement, les personnels des hôpitaux publics, des collectivités territoriales et des organismes de sécurité sociale. Ces 6,5 millions de salariés, protégés par de solides garanties collectives, constituent les deux tiers des syndiqués. Ils fournissent l'essentiel des permanents et des dirigeants syndicaux. Tout naturellement, leurs préoccupations et leur vision du monde imprègnent le mouvement syndical français ${ }^{7}$.

A l'opposé, on trouve les 16 millions de salariés employés dans le secteur privé. Parmi ces derniers, environ 4 millions disposent d'une présence syndicale sur leur lieu de travail. Dans quelques grandes entreprises de l'aéronautique, du pétrole, de la chimie, du livre ou de la presse, on trouve une situation proche de celles des entreprises publiques. Ailleurs, le syndicat se réduit à des élus invisibles en dehors des périodes électorales... Enfin pour les deux tiers des salariés du privé, le syndicalisme est devenue une idée vague et pas forcément sympathique. Ce qui amène à la seconde question : pourquoi ce divorce entre la majorité des salariés et les syndicats ?

\footnotetext{
${ }^{6}$ Pour un tableau complet : Andolfatto Dominique (dir.). Les syndicats en France. Paris : La Documentation française, 2004.

${ }^{7}$ Pour un tableau de synthèse : Dominique Andolfatto et Dominique Labbé. Sociologie des syndicats. Paris : La Découverte, 2000. Spécialement le chapitre IV.
} 


\section{Infidélité des adhérents ?}

L'étude des effectifs syndiqués permet également de démentir le mythe de la "passoire" syndicale. En effet, depuis longtemps, les analystes et les responsables syndicaux font état de "taux d'évaporation" considérables: annuellement, de 15 à $20 \%$, voire $25 \%$ des adhérents seraient partis et partiraient encore aujourd'hui. Ces chiffres sont contraires au bon sens : la population active salariée se renouvelle lentement et les nouveaux actifs, entrant chaque année sur le marché du travail, sont loin de pouvoir fournir un cinquième, voire un quart de l'effectif syndiqué total $(\mathrm{du}$ moins jusqu'aux années 1990). Faute de renforts frais, il faudrait admettre que les syndiqués français se comportent, depuis les années 1920, comme une armée d'opérette, démissionnant côté cour, pour ré-adhérer un peu plus tard côté jardin. Les archives syndicales disponibles ne font apparaître un tel turn-over que dans quelques cas très spécifiques (avant les années 1980) : établissements industriels employant surtout des OS, hôtellerie-restauration, etc. Si l'adhésion était assez brève (moyenne inférieure à 4 ans), c'est que la présence dans l'établissement l'était aussi. A l'inverse, la grande masse des syndiqués a été très fidèle. C'était d'ailleurs encore le cas au milieu des années $1990^{8}$.

La légende de la passoire est née de la manière suivante. Au PCF et à la CGT, bientôt imités par les autres confédérations, l'habitude s'est prise de publier, à espace régulier, le chiffre des adhésions nouvelles et des nouvelles bases (un peu à la manière des tableaux d'honneur stakhanovistes). Naturellement, ces chiffres étaient embellis et à l'heure du bilan annuel, la situation n'était généralement pas très brillante : sauf à reconnaître qu'on avait pêché par optimisme, il fallait inventer des "départs" pour compenser les adhésions "nouvelles" qu'on avait imprudemment claironnées.

Si cette fable de la passoire a été facilement acceptée, c'est qu'elle confère aux permanents syndicaux, seules figures stables au milieu de ce maelström de gens frivoles, le rôle de gardiens et de seuls représentants légitimes de l'organisation. Elle explique la faiblesse du mouvement syndical par l'inconscience, voire l'égoïsme des

\footnotetext{
${ }^{8}$ En 1993 deux sondages sur échantillons représentatifs tirés dans les fichiers de la CFDT montraient une ancienneté moyenne de l'adhésion égale à 18 ans pour les militants et à 15 ans pour les adhérents. Le turn-over est donc inférieur à 7\%. Voir Jean-Charles Basson et Dominique Labbé. Les militants de la CFDT et l'information syndicale (les lecteurs de Syndicalisme-Hebdo). Grenoble : CERAT-IEP. Dominique Labbé et Jean-Yves Sabot. Les adhérents de la CFDT et l'information syndicale (Les lecteurs de CFDT-Magazine). Grenoble : CERAT, décembre 1994,.
} 
salariés, pour lesquels les syndicalistes se dévouent comme ils peuvent. Ainsi se trouvent justifiées les aides multiples apportées aux syndicats.

Le constat est donc le suivant : à partir de 1976-78 et en moins de 10 ans, la majorité des adhérents ont quitté les syndicats. Grâce à plusieurs enquêtes menées entre 1983 et 1988, ces "désyndiqués" sont assez bien connus ${ }^{9}$. En moyenne, ils cotisaient depuis plus d'une dizaine d'années. Autrement dit, il s'est produit un sérieux divorce. Comme toujours dans les séparations, plusieurs facteurs se sont cumulés.

Les raisons politiques, propres à cette période, portent la principale responsabilité dans près de 4 départs sur 10 : désunion de la gauche puis déceptions de l'alternance, reprise en main de la CGT par le PCF, chasse aux "coucous" gauchistes dans la CFDT, polémiques publiques entre ces organisations autrefois alliées, prise de contrôle de certaines organisations FO par les trotskistes lambertistes...

L'autre cause essentielle du divorce est plus inattendue : non seulement les syndicalistes n'ont guère recruté mais ils se sont aussi débarrassés de certains adhérents. En effet, plus du tiers des anciens adhérents que nous avons rencontrés seraient volontiers restés syndiqués. On ne venait pas leur demander la cotisation, la section ne se réunissait plus, il n'y avait plus de permanence, personne ne répondait aux courriers ou aux coups de fil. Or, dans la plupart des établissements concernés, la section syndicale continuait bel et bien d'exister. Elle présentait des listes aux élections et elle disposait d'élus. Autrement dit, non seulement les responsables n'ont pas cherché de nouveaux adhérents, mais ils se sont désintéressés d'une partie des syndiqués par négligence, ou parce que ces anciens étaient en désaccord politique avec eux, ou parce qu'ils représentaient des "coûts" jugés prohibitifs (en temps de délégation pour des interventions individuelles). En quelque sorte, ces responsables ont volontairement choisi la voie d'un certain malthusianisme.

Le mouvement commence avec la création, en décembre 1968, du délégué syndical (DS) nommé par le syndicat. Cette fonction a été systématiquement cumulée avec les mandats de DP ou d'élu au comité d'entreprise (CE). Comme les fonctions de DS ou d'élu CE sont plus gratifiantes, les heures délégation attribuées au DP ont été utilisées à autre chose et le DP a disparu des ateliers et des bureaux.

\footnotetext{
${ }^{9}$ Synthèse dans : Dominique Labbé et Maurice Croisat. La fin des syndicats ? Paris : L'Harmattan, 1992. Ces anciens syndiqués avaient appartenu à des syndicats dont nous connaissions précisément l'histoire afin de pouvoir contrôler les dires des enquêtés.
} 
Le mouvement s'est amplifié avec les lois Auroux (1982) qui ont octroyé, à la section syndicale et au CE des moyens financés par l'employeur. Alors, les syndicalistes se sont demandé : à quoi bon se donner du mal pour collecter de maigres cotisations alors que nous avons tout ce qu'il nous faut pour fonctionner sans avoir à jouer les "assistantes sociales" ?

Telle est la raison pour laquelle la désyndicalisation coïncide à peu près partout avec la mise en oeuvre de nouveaux moyens attribués aux syndicats par la loi, les administrations et les entreprises. Certes, auparavant, le syndicat semblait bien précaire, surtout s'il ne contrôlait pas le CE, et il a semblé logique de lui donner une assise plus solide et une meilleure visibilité dans l'entreprise ou dans les administrations. Pourtant, la création de la section syndicale, ou l'octroi de droits syndicaux correspondants dans les administration, loin de renforcer les syndicats, les a finalement affaiblis en les transformant en institutions ${ }^{10}$ lointaines et étrangères aux salariés.

La faiblesse du recrutement conduit logiquement à un syndicalisme sans adhérent. En effet, il existe, chaque année, une "évaporation" incompressible d'au moins 5\% des syndiqués : c'est en moyenne, le nombre d'événements imparables - décès, longue maladie, accident, chômage, déménagement... - qui se traduisent par autant de démissions apparentes. C'est pourquoi, après les départs massifs des années 1978-88, la plupart des syndicats se sont lentement rétrécis à une poignée d'élus et de mandatés.

Ceci amène à examiner de plus près les moyens dont disposent les syndicats.

\section{Faiblesse des moyens?}

Personne n'a cherché à connaître précisément les moyens dont disposent les syndicats. Il semble même y avoir une sorte de consensus pour minimiser les choses ou pour les laisser dans le flou. Le rapport, remis au Premier ministre par R. HadasLebel au printemps 2006, en offre une nouvelle illustration ${ }^{11}$. Ce document dresse une liste assez complète des principales ressources mais il donne très peu de chiffres.

\footnotetext{
${ }^{10}$ Cette institutionnalisation des syndicats a été notée pour la première fois par Gérard Adam (Le pouvoir syndical. Paris : Dunod, 1983).

${ }^{11}$ Raphaël Hadas-Lebel. Pour un dialogue social efficace et légitime : représentativité et financement des organisations professionnelles et syndicales. Rapport au Premier ministre. Paris : La Documentation française, mai 2006.
} 
Ces quelques chiffres sont souvent inférieurs à la réalité et aucune totalisation n'est tentée. Paradoxalement, le rapporteur semble mieux renseigné sur les ressources des syndicats... scandinaves allemands, italiens ou belges !

On peut classer les ressources, autres que les cotisations, en trois groupes (par ordre décroissant d'importance) : les mises à disposition de personnels qui continuent à être payés par leur employeur antérieur ; les prélèvements sur les organismes paritaires et, enfin, les subventions publiques.

Les personnels mis à disposition au titre des "droits syndicaux" représentent la ressource la plus importante. Ils se consacrent, partiellement ou totalement, au syndicat, tout en continuant à percevoir leur traitement ou leur salaire et tout en bénéficiant d'une promotion au moins égale à celle de la moyenne de leurs collègues de même grade ou de même qualification ${ }^{12}$. Nous avons déjà évoqué les DS dans les entreprises soumis au droit commun (20 heures par mois). Personne ne s'est préoccupé d'en connaître le nombre et les affiliations syndicales.

En ce qui concerne les fonctionnaires, le rapport Hadas-Lebel donne quelques chiffres (généralement revus à la baisse par rapport aux déclarations antérieures) et il omet les administrations les plus généreuses comme la police (sur les 140000 policiers, 1600 disposent d'une décharge totale de service au titre des "droits syndicaux") ou La Poste France-Télécom (3 500 équivalents temps plein).

Les grandes entreprises publiques sont au moins aussi généreuses que l'Etat. Par exemple, le rapport Hadas-Lebel indique que, pour la SNCF, ces mises à disposition représentent l'équivalent de 590 temps pleins. Cette manière de présenter les choses est partielle. Il faut ajouter les délégués syndicaux (dans les entreprises publiques, les hôpitaux, les caisses de sécurité sociale) et les autorisations d'absence en faveur des membres d'un organe directeur - lorsque les réunions de cet organisme ont lieu durant le temps de travail - ou pour la participation aux congrès syndicaux, pour la formation sociale et syndicale, etc. La direction de la SNCF n'a jamais fait mystère que l'ensemble des dispositions relatives au seul droit syndical représente $1 \%$ de la masse salariale, soit 1700 équivalents temps plein ${ }^{13}$. Naturellement, il faut encore ajouter les

\footnotetext{
${ }^{12}$ A EDF, par exemple, un dispositif ad hoc a été mis en place qui garantit l'évolution automatique de carrière des «mandatés ».

${ }^{13}$ Pourquoi le rapport Hadas-Lebel ne fait-il pas état de ce dernier chiffre alors que la direction générale de l'entreprise et les syndicats ne le cachent pas ? Serait-ce parce que ce chiffre va à l'encontre de la thèse implicite du rapport selon laquelle il faut augmenter les aides aux syndicats ?
} 
délégués du personnel et les élus CE mais, normalement, le syndicat n'est pas leur tâche principale.

Parmi les entreprises aussi généreuses que la SNCF : EDF-GDF, la RATP, AirFrance, Aéroport de Paris... Au total, près de 7000 équivalents temps plein pour les seules entreprises publiques.

Les collectivités territoriales (régions, départements, communes...) et les hôpitaux publics ont adopté des systèmes semblables et parfois plus généreux. Par exemple, la largesse de la ville de Marseille est proverbiale.

Les principales caisses de sécurité sociale agissent de même à deux niveaux. D'une part, pour leur propre personnel, elles accordent assez libéralement des mises à disposition et des décharges de service en fonction des résultats aux élections dans leurs établissements, selon un système inspiré de celui qui vient d'être présenté. D'autre part, de manière discrète, elles fournissent aux syndicats et au patronat, des "conseillers techniques" qui sont censés les assister dans leur fonction de "gestionnaires" des caisses de sécurité sociale.

Il est impossible de connaître le total des heures ainsi mises à disposition des syndicats. Mais, en dehors de quelques cas aberrants, comme la police nationale, le ratio se situe entre $0,7 \%$ et $1 \%$ du total des emplois. En adoptant le pourcentage le plus faible $(0,7 \%)$, on peut estimer que, au total, dans les trois fonctions publiques, les organismes de sécurité sociale, les grandes entreprises nationales, les seuls "droits syndicaux" - décharges de service, partielle ou totale, autorisations d'absence, etc. représentent l'équivalent de 40000 emplois temps plein ${ }^{14}$. La réalité est certainement supérieure à ce minimum.

Ce ne serait peut-être pas cher payer si ces organismes y gagnaient en efficacité, si l'ambiance au travail était meilleure et l'absentéisme négligeable. Est-ce le cas ? Dans ce vaste secteur public et para-public, les rapports sociaux et les conditions de travail se sont-ils améliorés depuis près de 40 ans que cette politique libérale a été lancée ? D'ailleurs, s'est-on préoccupé de savoir ce que font réellement ces mis à disposition ?

Certes, on peut objecter que ce contingent d'heures est à peine plus généreux que ce que la loi accorde aux représentants élus du personnel dans les entreprises privées

\footnotetext{
${ }^{14}$ En prenant pour base les seuls titulaires - inscrits sur les listes électorales des commissions administratives paritaires des trois fonctions publiques et des CE des entreprises publiques. En réalité, ce vaste secteur "public" représente, avec les fonctionnaires stagiaires et les contractuels, plus de 6 millions de salariés.
} 
de plus 50 salariés. Mais ce n'est pas la même chose d'accorder quelques heures à des gens élus par leurs collègues de travail et de laisser une direction syndicale décider arbitrairement que tel ou tel salarié sera extrait de son milieu professionnel pour être affecté à un poste qui n'a souvent aucun rapport avec son entreprise ou son administration. Comme la direction syndicale peut révoquer la personne mise à disposition sans fournir d'explication, les conséquences sont faciles à imaginer : le syndicaliste mis à disposition vit, le regard tourné vers le secrétaire général, guettant les signes de faveur ou de défaveur, dans un univers de grisaille où la parole est contrainte et les votes acquis à l'avance.

En tous cas, au seul titre du secteur public et para-public, les syndicats français disposent de beaucoup plus de permanents que n'importe quel autre syndicat équivalent dans le monde. Par exemple, la fédération allemande des syndicats de la fonction publique et des transports comptait 1,8 million d'adhérents à la fin des années 1990 et disposait de 2600 permanents qu'elle salariait presque tous elle-même. Autrement dit, avec le même effectif que tous les syndicats français réunis, cette organisation, relativement prospère, disposait d'à peine plus du vingtième des moyens en personnel de ses homologues françaises...

La seconde catégorie de ressources (en importance) provient des prélèvements opérés sur les organismes paritaires.

L'exemple le mieux connu est celui de la formation permanente des adultes. Il y a en France une centaine d'organismes paritaires collecteurs des fonds de la formation permanente (OPCA) et environ 600 organismes collecteurs de taxe d'apprentissage (on ne sait rien de ces derniers). Les OPCA retiennent 10 à 12\% des sommes collectées, pour "frais de gestion", et en redistribuent officiellement $0,75 \%$ - soit environ 20 millions d'euros en 2001 - aux partenaires sociaux fondateurs de l'organisme (les fédérations professionnelles adhérentes aux 5 confédérations représentatives, plus le Medef, la CGPME et l'UPA). A cela, il faut ajouter les mises à disposition de locaux, de conseiller(s) technique(s), la location (au prix fort) de stands lors des congrès syndicaux, l'achat d'espaces publicitaires dans la presse syndicale, les contrats d'étude, etc. Ces sommes n'apparaissent pas dans les budgets publiés par les confédérations et les fédérations. Elles sont logées dans des satellites dont les comptes sont inconnus.

En 1996, pour coiffer l'ensemble, un accord national interprofessionnel a créé le FONGEFOR qui reçoit aussi $0,75 \%$ de la collecte globale de tous les OPCA, soit, en 
2001, environ 20 millions d'euros qui ont été partagés par moitié entre les confédérations patronales et syndicales. Autrement dit, par ce seul canal, chaque confédération a reçu directement 2 millions d'euros et 2 autres millions ont été versés aux différentes fédérations du privé par les OPCA. Pour cette même année 2001, le total des cotisations touchées par les confédérations sont, pour la CFDT, de 5,5 millions d'euros et, pour la CGT, d'un peu moins de 4 millions d'euros. Autrement dit, la signature des accords concernant la seule formation permanente, largement préparés par l'administration, et la participation à quelques discussions autour de son actualisation, apportent à peu près autant d'argent aux confédérations que tous leurs adhérents.

Il ne s'agit pas d'un cas isolé mais d'un système général. Dès qu'elles sont gérées de manière "paritaire", les caisses de retraite complémentaire, les caisses de sécurité sociale nationales et régionales (maladie, allocations familiales, chômage...), les mutuelles, versent des sommes souvent considérables aux partenaires sociaux qui siègent à leurs conseils d'administration. Dès que l'organisme atteint une certaine taille, il met aussi à leur disposition des "conseillers techniques" qui sont autant de permanents syndicaux et qui, pour la plupart, n'apportent aucune contribution à l'activité de l'organisme qui les salarie.

Le contrôle de l'IGAS (Inspection générale de la sécurité sociale) sur ces organismes étant fort discret, il est impossible de chiffrer même grossièrement les ressources qu'y prélèvent les organisations syndicales et patronales. On peut d'ailleurs avoir des doutes sur la qualité de la gestion de certains de ces organismes - tout particulièrement dans le cas de la formation des adultes, trop éclatée, coûteuse, peu efficace et fortement inégalitaire - et ajouter que la plupart de ces organismes n'ont plus de "paritaire" que le nom, paravent commode pour une gestion de plus en plus étatisée $^{15}$. Quand, de plus, ces organismes sont contraints de faire des économies, d'augmenter les cotisations et de réduire les prestations, l'obscurité de la gestion et les charges indues deviennent choquantes. Enfin, le "budget" sanitaire et social de la nation représentant une fois et demi celui de l'Etat, l'opacité et souvent le laxisme de la gestion sont évidemment inquiétants.

\footnotetext{
${ }^{15}$ Nezosi Gilles. «La crise du paritarisme ». Problèmes politiques et sociaux, $\mathrm{n}^{\circ} 844,8$ septembre 2000.
} 
La troisième ressource (en importance) est constituée par les subventions - en espèce ou en nature - versées par les collectivités publiques et par les entreprises.

Personne n'a cherché à en dresser un tableau d'ensemble, ne serait-ce que pour le seul budget de l'Etat. On sait que, en 2005, le ministère du Travail a versé à l'ensemble des organisations syndicales 29 millions d'euros, pour financer la formation des délégués syndicaux, élus du personnel, conseillers prud'hommes. A ce seul titre, la confédération CGT a reçu : 7,5 millions d'euros (soit 1,8 fois plus que le total des cotisations encaissées par la confédération). On connaît également la subvention versée par le ministère de la Fonction publique au titre de la formation des membres des commissions paritaires de la fonction publique : 363000 euros pour chacune des 5 organisations les plus "représentatives" (CGT, CFDT, FO, UNSA, FSU) et la moitié aux deux autres (CFTC, CGC). Ces sommes n'apparaissent pas dans les budgets des confédérations, elles sont logées dans des satellites chargés de la formation, comme par exemple l'Institut Benoît Frachon pour la CGT ou l'Institut Belleville pour la CFDT.

Les administrations mettent également à disposition des syndicats des moyens matériels (locaux et moyens de fonctionnement, plus le matériel informatique).

Aux subventions de l'Etat, il faut ajouter les subventions équivalentes versées par les régions, les départements et quelques grandes villes. De plus, la plupart des villes grandes et moyennes fournissent aussi des locaux (les bourses du travail) dont elles prennent en charge les frais de chauffage, gardiennage, nettoyage, etc.

On peut enfin ajouter les reversements effectués par les membres du conseil économique et social (comme les députés versent une certaine part de leurs indemnités à leur parti). Par exemple, la confédération CFDT affiche à ce titre une recette de 580000 euros en 2004. Mais l'on ne sait rien des sommes en provenance des conseils économiques et sociaux régionaux.

A ce simple niveau des subventions publiques, il est certain que le total des versements et des aides indirectes est sans commune mesure avec les cotisations des membres.

Pour les entreprises privées, les syndicats représentatifs disposent, depuis 1982, de locaux dans les établissements de plus de 50 salariés. Beaucoup d'entreprises prennent en charge les frais de fonctionnement (téléphone, polycopie, matériel informatique...). Les syndicats qui dirigent un CE disposent de la subvention de 0,2\% de la masse salariale que l'entreprise doit verser pour l'administration du comité. Les 
entreprises prennent aussi en charge les congés de formation des syndicalistes (que la loi fixe au minimum de 0,08 pour mille de la masse salariale, mais il y a des accords d'entreprise plus généreux). En revanche, les entreprises privées accordent plus rarement des décharges de services. Lorsque l'accord d'entreprise prévoit des droits syndicaux plus généreux que le minimum légal (ce qui est de plus en plus fréquent), beaucoup de directions préfèrent verser au syndicat une somme équivalente aux heures en question plutôt que de désorganiser les services.

Enfin, certaines entreprises se débrouillent pour subventionner "leurs" syndicats : location de stands lors des congrès, publicités dans les revues syndicales, contrats d'étude etc. Certains groupes prennent en charge la cotisation grâce au "chèque syndical" instauré notamment par AXA ${ }^{16}$, le GAN, Casino...

Naturellement, toutes ces aides sont difficiles à chiffrer mais elles sont considérables. Par exemple, au début des années 1990, les seules sommes d'argent versées par l'entreprise représentaient les deux tiers du budget de la section CGT de RVI-Vénissieux et $90 \%$ de celui de la section de Sollac (Fos-sur-mer) ${ }^{17}$. Une part variable, mais impossible à connaître, est reversée aux fédérations et aux confédérations - parfois sous forme d'achat de timbres.

Autrement dit, dans les entreprises privées, les syndicats peuvent très bien fonctionner, sans avoir besoin de se préoccuper des cotisations. Dans ces conditions, on peut se demander quelle est l'indépendance réelle des sections syndicales vis-à-vis des employeurs. Cette question est d'autant plus importante qu'en France, le dialogue social est censé être trop faible.

\footnotetext{
${ }^{16}$ Le rapport Hadas-Lebel indique que moins de la moitié des salariés d'Axa utilisent ce chèque (Opp. cit., p. 113). Indifférence ? hostilité envers cette subvention patronale?

${ }^{17}$ Laurent Olivier. La fédération CGT de la métallurgie de la Libération à nos jours. Organisation et syndicalisation. Annexe au rapport d'enquête pour le ministère du travail. Grenoble : CERAT, 1996.
} 


\section{Faiblesse du dialogue social ?}

Quantitativement, cette opinion commune est fausse. En effet, dans aucun autre pays, les salariés ne disposent d'une telle "couverture" conventionnelle ${ }^{18}$. Pour le secteur privé et les établissements publics à caractère industriel et commercial, 97\% des salariés, travaillant dans des établissements d'au moins 10 salariés, sont "couverts" par des accords collectifs et l'on approcherait les $90 \%$ pour les entreprises de moins de 10 (grâce au développement des accords touchant l'artisanat et les petits commerces). Même chez les champions du monde de la syndicalisation (Finlande, Suède ou Danemark, où $80 \%$ des salariés sont syndiqués), on ne parvient pas à de tels taux et de très de loin!

De même en ce qui concerne les entreprises et les établissements, le nombre des accords place la France très haut dans la hiérarchie des pays les plus "conventionnels": cette négociation concerne directement près de 5 millions de salariés du secteur privé (soit près d'un sur trois) et pas loin de la moitié des établissements de 50 salariés et plus qui sont théoriquement astreints à une négociation annuelle. Là encore, la plupart des grands pays industriels ne connaissent pas de pareils taux. Il est vrai que, en France, tout est fait pour faciliter cette négociation : depuis 1995, en l'absence de section syndicale, les élus du personnel, voire un salarié "mandaté" par une organisation syndicale peuvent signer des accords qui seront réputés avoir reçu l'approbation des salariés de l'entreprise.

Les enjeux de cette négociation sont de plus en plus importants. L'aménagement du temps de travail occupe la première place plus ou moins à égalité avec les salaires. La plupart du temps, il ne s'agit pas de réduire le temps de travail, mais de rendre la main d'œuvre flexible : annualisation du temps de travail, fractionnement de la journée de travail et des congés annuels, rémunération des cadres au forfait, récupération des journées chômées mais non fériées, travail en équipe, astreintes... Pour les salaires, l'individualisation, notamment des primes, est devenu un thème majeur. D'autres

\footnotetext{
${ }^{18}$ Les chiffres concernant la négociation collective sont tirés des bilans annuels présentés par le ministre du Travail, à la fin du mois de juin, devant la Commission nationale des conventions collectives (autrefois publiés par la Documentation française et maintenant placés en ligne sur le site du ministère du Travail). Voir également : Dominique Labbé et Gilles Nezosi. "Négociation collective, paritarisme et démocratie sociale". In Dominique Andolfatto (dir). Les syndicats en France. Paris : La Documentation française, 2004.
} 
thèmes sont en forte augmentation comme l'emploi (les procédures de réduction $\mathrm{du}$ personnel) ou la naissance d'un système d'épargne et de prévoyance.

Contrairement à ce que laisse entendre le discours sur le "blocage" de la société française, on assiste à une "révolution silencieuse" dans les entreprises : le droit du travail est progressivement assoupli - voire démantelé sur certains points comme le temps de travail - grâce à la multiplication des conventions dérogatoires.

Cette révolution se fait-elle en faveur des salariés? Plusieurs indices permettent d'en douter.

La "propension à signer" est l'un de ces indices. Ce taux est calculé de la manière suivante : par étiquette syndicale, on place le nombre d'accords signés au numérateur et, au dénominateur, le nombre de négociations où le syndicat était présent. On s'attendrait à ce qu'il y ait des différences significatives entre organisations. Or, au niveau des entreprises, il n'y en a pas : pour l'année 2004, la propension à signer se situe entre $84 \%$ (CGT) et 92\% (CFE-CGC). Cela signifie que, en dehors de quelques très grandes entreprises, souvent du secteur public, la CGT comme FO signent autant que les autres syndicats. En réalité, les syndicalistes n'ont guère le choix. D'une part, comment le DS pourrait-il s'opposer à son patron alors que son statut de salarié le place sous l'autorité de celui-ci et qu'il est dépendant du directeur des ressources humaines pour sa carrière? D'autre part, comment refuser des accords qui prévoient des "droits syndicaux", plus favorables que la simple application des lois et conventions de branche, notamment en ce qui concerne les heures du DS, voire sa mise à disposition?

A l'opposé, les supposés bénéfices qu'en tirent les salariés ne sont pas évidents. De ce point de vue, l'évolution des conditions de travail fournit l'indice le plus intéressant. Dans le passé, l'action syndicale a été un puissant facteur d'amélioration de ces conditions. Par exemple, on lui doit l'invention des délégués à la sécurité dans les mines, institution qui inspirera les comités d'hygiène et de sécurité. Mais la loi et les inspecteurs du travail ne peuvent à eux seuls rendre effectives ces institutions. Pour se traduire dans la réalité, il leur a fallu un réseau collectif capable d'alerter les délégués, quand se pose un problème, et de contrôler ensuite que les décisions sont appliquées. Sans action collective, ces institutions de prévention deviennent des coquilles vides. Pire, les élus, surtout préoccupés par leur réélection, se mettent d'accord avec l'employeur pour compenser les "pénibilités" ou les "nuisances" par des primes, électoralement payantes, mais catastrophiques pour la santé et la sécurité des salariés. 
En France, on assiste depuis 25 ans à une dégradation continue des conditions de travail de la majorité des salariés du secteur privé, à une augmentation inquiétante des accidents du travail et des maladies professionnelles. Cette dégradation est confirmée par toutes les enquêtes ${ }^{19}$. Pourtant, ces mauvaises nouvelles sont tombées dans une indifférence générale : les pouvoirs publics et le patronat sont restés silencieux, de même que les confédérations syndicales, la presse s'en est désintéressée, les chercheurs ont cherché ailleurs, à quelques rares exceptions qui n'ont pas été écoutées.

Tout se passe comme s'il y avait eu un échange inégal. Pour les salariés : une réduction (assez faible) du temps de travail, et quelques primes, en contrepartie d'une intensification et d'une flexibilité du travail souvent brutale ${ }^{20}$. L'affaiblissement de la capacité d'action collective - dont le syndicat était le principal vecteur - fait que, pour la première fois dans l'histoire industrielle moderne, les employeurs ne se heurtent pas à la résistance des opérateurs qui était la hantise du taylorisme. Au contraire, la disparition des militants et de l'ambiance de solidarité, l'éclatement des équipes traditionnelles en modules autonomes ont laissé les salariés démunis ${ }^{21}$. L'individualisation des tâches est devenue la règle et elle fait peser sur chaque salarié un autocontrôle autrement plus rigoureux que celui du "bureau des méthodes" d'autrefois. Sans doute que les salariés n'accepteraient pas de revenir au travail à la chaîne, ni à l'organisation pyramidale d'antan, mais peu ont conscience du prix dont ils paient une autonomie (souvent illusoire).

La principale question est finalement la suivante : les salariés se sentent-ils engagés par les accords signés en leur nom ? Depuis 25 ans, les sondages indiquent que la majorité des salariés n'ont pas confiance dans les syndicats pour la défense de leurs

\footnotetext{
19 Michel Gollac et Serge Volkoff, Les conditions de travail, Paris, La Découverte, 2000. Philippe Askenazy. Les désordres du travail, Paris, Le Seuil, 2004. Signalons toutefois : CFDT. Le travail en question : enquêtes sur les mutations du travail, Paris, Syros-La Découverte, 2000 (les dirigeants de la CFDT n'ont guère donné d'écho à ce document). Certains résultats des enquêtes sur les conditions de travail ont été publiés par la Direction de la recherche du ministère du Travail dans Premières informations (accessibles en ligne : www.travail.gouv.fr/). Voir également, les enquêtes européennes en ligne sur : www.fr.eurofound.eu.int/.

${ }^{20}$ Il s'agit d'un mouvement mondial mais avec des particularités françaises. Voir par exemple : Howard Gospel and Andrew Pendleton (eds.). Corporate Governance and Labour Management: An International Comparison. Oxford: Oxford University Press, 2005. Spécialement : Michel Goyer and Bernard Hancke. "Labour in French Corporate Governance: The Missing Link", op. cit., p. 173-196.

21 Voir par exemple : Stéphane Beaud et Michel Pialoux. Retour sur la condition ouvrière. Enquête aux usines Peugeot de Sochaux-Montbéliard. Fayard, Paris : 1999.
} 
intérêts. L'actualité donne souvent l'exemple de salariés en grève contre des dispositions négociées avec "leurs" syndicats.

$\mathrm{Au}$ fond, pour la majorité des dirigeants d'entreprise ou des administrations publiques, la négociation est un mal nécessaire pour être formellement "en règle" avec la loi et avec l'administration du travail. Comparée à l'Allemagne, aux pays scandinaves et à l'Amérique du nord, la négociation à la française tient du simulacre. L'administration et les employeurs fixent les thèmes et l'agenda, apportent les projets et établissent le relevé des conclusions. D'ailleurs, combien d'employeurs parlent de "leurs" syndicats ou de "leurs" partenaires sociaux ? Cela explique le scepticisme des principaux intéressés : les salariés (ou les assurés sociaux, pour ce qui concerne la réforme de l'Etat providence). D'où les propositions tendant à "renforcer" les syndicats.

\section{Représentativité et financement public}

Deux propositions sont habituellement avancées pour renforcer les syndicats :

- organiser des "élections de représentativité" qui permettront aux salariés d'une branche économique - en votant tous le même jour, ou dans une brève période de temps, pour des listes présentées par les organisations les plus représentatives - de choisir les appareils syndicaux, chargés de parler en leur nom avec les employeurs de la branche en question ${ }^{22}$. On attend de ces élections - calquées sur les législatives qu'elles confèrent aux négociateurs syndicaux une légitimité moins formelle que la présomption légale de représentativité. On espère ainsi légitimer aux yeux des salariés les conventions signées en leur nom ;

- financer les syndicats en fonction du nombre de voix obtenues à ces élections suivant le système adopté pour financer les partis politiques.

Si beaucoup de détails sont discutés, il en est deux sur lesquels l'accord semble fait : les bureaux de vote seront situés sur le lieu du travail et le scrutin se déroulera pendant les heures de travail. C'est reconnaître que, si les bureaux de vote étaient situés en dehors des établissements, personne n'irait voter. La montée de l'abstention aux élections des conseillers prud'hommes est là pour le prouver : les syndicats sont aujourd'hui incapables de mobiliser les salariés pour les amener aux urnes en dehors

\footnotetext{
${ }^{22}$ Voir par exemple, Raphaël Hadas-Lebel. Pour un dialogue social... Op.cit., p. 88-92.
} 
du lieu de travail, même quand ces salariés connaissent parfaitement l'utilité de l'institution.

Mais comment organiser un tel scrutin, à peu près en même temps, dans les centaines de milliers d'entreprise du commerce, de l'hôtellerie-restauration, du bâtiment ou de la réparation automobile ? Qui va établir les listes électorales ? Qui va contrôler que les salariés peuvent aller voter ? Qui va surveiller les urnes ? Qui va veiller à la régularité du dépouillement? Qui va centraliser les résultats ? En tout cas, on ne peut compter sur les syndicats puisqu'ils sont absents de la plupart des entreprises privées françaises, y compris dans la majorité des établissements de 50 salariés et plus ${ }^{23}$.

A ce propos, on remarquera que les salariés votent déjà beaucoup mais que, à part les élections aux commissions paritaires, dans les trois fonctions publiques, les résultats sont mal connus : aucune statistique concernant les sections syndicales ni les élections des DP (les plus importantes), données discutées concernant les élections aux CE et aux délégations uniques.

Va-t-on confier une nouvelle mission impossible à l'administration du Travail ? En effet, l'Inspection du Travail semble à la dérive, incapable d'assumer sa mission première de surveillance des entreprises, perdue dans un maquis de plusieurs centaines de "dispositifs pour l'emploi" - que chaque gouvernement a empilé sur ceux des précédents - et dont elle ne parvient pas à contrôler l'exécution. Il serait absurde d'ajouter une mission supplémentaire à toutes celles que l'Inspection du Travail ne parvient plus à assumer.

De toute façon, ces élections, calquées sur un modèle politique, n'apporteront aucune solution au problème essentiel : la perte de légitimité des organisations syndicales. Cette perte de légitimité provient de leur disparition des lieux du travail et de l'absence de services individuels aux salariés. Ce n'est pas une campagne électorale de plus qui arrangera les choses.

Dès lors, avant d'ajouter une nouvelle subvention, à celles déjà considérables que reçoivent les syndicats, ne faudrait-il pas mettre à plat l'ensemble de leurs moyens ? Sinon le scepticisme de l'opinion pourrait se transformer en colère. En effet, comment

\footnotetext{
${ }^{23}$ En l'absence de statistique sur les sections syndicales, on est réduit à des estimations. Du début des années 1980 à 1993, le recul des implantations syndicales a été rapide (Labbé Dominique. Les élections aux comités d'entreprise (1945-1993). Grenoble : CERAT, 1995). Depuis lors, cette mesure n'est plus possible mais le sens de l'évolution ne fait pas de doute.
} 
justifier que l'on accorde encore plus d'aides à des bureaucraties, déjà largement entretenues par les salariés, les contribuables, les assurés sociaux et les entreprises à qui l'on demande sans cesse de nouveaux sacrifices?

Plus largement, on peut se demander s'il n'y a pas, en France, une véritable obsession de la subvention. Par exemple, on a introduit dans le code du travail la possibilité, pour le salarié d'une entreprise dépourvue de représentation syndicale et d'élu du personnel, de se faire assister par un "conseiller du salarié". L'idée est excellente mais pourquoi les salaires, charges et frais de transport de ce conseiller doivent-ils être pris en charge par les collectivités locales ? Pourquoi ce droit ne serait-il pas financé par la caisse de résistance du syndicat (ou par des associations d'entraide si le salarié ne veut pas faire appel à un syndicat) ? Pourquoi le contribuable devrait-il se substituer à une action collective que l'on considère a priori comme impossible, alors que notre histoire sociale fourmille d'exemples en sens contraire?

\section{Conclusions}

Depuis les années 1960, les gouvernements successifs et les chefs des grandes entreprises ont cherché à renforcer le syndicalisme, car ils considéraient que des syndicats forts (raisonnables ?) étaient des acteurs indispensables au dialogue social et à la modernisation des entreprises ou des administrations. Pourtant le résultat est à l'opposé du but apparemment recherché : affaiblissement continu des organisations, divisions infinies, représentativité de plus en plus douteuse et montée des blocages de tous ordres... Les multiples droits, protections, aides qui ont été accordés aux syndicats auraient dû bénéficier à l'ensemble des salariés. Dans les faits, ils ont été privatisés au profit de quelques dizaines de milliers de professionnels de la représentation - les syndicalistes - qui n'ont plus de liens, autres qu'électoraux, avec les salariés qu'ils sont censés représenter et une connaissance très théorique de leur situation réelle et de leurs attentes.

Comment sortir de cette impasse?

Outre la révision de la "représentativité" et la transparence des ressources des syndicats, le tableau qu'on vient de dresser suggère quelques pistes.

La première consiste à replacer le salarié au centre de l'activité syndicale : la fonction essentielle d'un syndicat est d'aider le syndiqué à résoudre ses problèmes personnels sur le lieu du travail. Pour cela, il est nécessaire de limiter le cumul des 
mandats de DP, de DS et d'élu au CE et de restaurer la fonction de DP, fonction essentielle pour l'amélioration des relations sociales sur le lieu du travail et pour la relance de la syndicalisation.

La seconde piste consiste à rééquilibrer la négociation collective, notamment dans les entreprises, en remplaçant le DS par un négociateur professionnel, appointé par le syndicat, et en interdisant toute subvention directe ou indirecte en provenance des entreprises et des administrations. Il semblerait également logique que, avant de s'asseoir à la table des négociations, les syndicats apportent la preuve qu'ils ont une proportion suffisante d'adhérents dans les entreprises concernées et que les salariés puissent ratifier les accords signés en leur nom.

Mais le souhaite-t-on réellement?

Du côté des syndicats, ce n'est pas le cas. Les syndicats français sont prospères et fonctionnent pratiquement sans adhérents. Ils sont contrôlés par des professionnels qui ne souhaitent pas que les adhérents deviennent le centre de l'activité syndicale ou que la lumière soit faite sur leurs ressources. Cependant, le silence qui entoure l'importance réelle de leurs moyens constitue une sorte d'aveu.

Du côté des entreprises et des administrations, on s'accommode aussi fort bien de la situation actuelle. Au fond, les grandes entreprises françaises, les administrations centrales, les institutions de sécurité sociale, les hôpitaux ou les principales collectivités locales fonctionnent de manière assez semblable. Leurs dirigeants sont issus des mêmes écoles et des mêmes corps. D'ailleurs, dans les états-majors des grandes entreprises, on parle de "nomination", de "cabinet", etc. Pour les choses importantes, ces chefs procèdent comme Alexandre avec le nœud gordien. Tout au plus, reçoit-on, au préalable et en grande pompe, quelques chefs syndicaux pour mimer une concertation de façade. La méthode a d'autant plus de chance de réussir que l'on aura, au préalable, privé ceux sur qui tombe le glaive des moyens d'une action collective et que l'on se sera assuré l'appui de l'un ou l'autre des "représentants" de ces victimes (ou de leur complicité passive). Les multiples aides et protections accordées aux syndicats ne servent-elles pas à cela ? 